\title{
Kinematic Models For Pitch Location Metrics in Professional Baseball Pitchers
}

\author{
Joseph E Manzi, BS ${ }^{1 *}$, Spencer Krichevsky, $M S^{2}$, Nicholas Roberts, $M P H^{1}$, Ryan C Rauck, MD \\ and Joshua S Dines, $M D^{4}$
}

${ }^{1}$ Weill Cornell Medical College, New York, NY, USA

${ }^{2}$ Stony Brook University, Department of Biomedical Informatics, Stony Brook, NY, USA

${ }^{3}$ Department of Orthopaedics, The Ohio State University Wexner Medical Center, Columbus, OH, USA

${ }^{4}$ Sports Medicine Institute Hospital for Special Surgery, New York, NY, USA

\begin{abstract}
Consistency and accuracy in pitch location is paramount for professional baseball pitchers, however, the contribution of individual kinematic parameters is poorly understood. Professional baseball pitchers $(n=322)$ were instructed to throw 8-12 fastballs while assessed with motion-capture technology $(480 \mathrm{~Hz})$; pitch location was tracked by coaching staff. Elastic net regression with automated parameter optimization was utilized to model the consistency and accuracy of each pitch with a random forest model implemented to assess feature importance. Trunk tilt at foot contact (FC) was the most significant predictor of accuracy indicated by a percent increase of the mean square error (6.6\% MSE), followed by lead hip flexion at FC ( $4.2 \% \mathrm{MSE})$, shoulder abduction at FC (4.2\% MSE), and trunk tilt at ball release (BR) (3.8\% MSE). For pitch consistency, the resulting model explained $57 \%$ of the variance. Trunk tilt at FC had the highest predictive value (3.7\% MSE), followed by trunk flexion at BR (3.3\% MSE), foot rotation at FC (3.2\% MSE), and back hip flexion at FC ( $2.6 \% \mathrm{MSE})$. Four of the top six parameters found to be predictive in both models involved variance at the hip and trunk. Pitchers should strive for consistency in hip and upper torso motions, particularly at foot contact.
\end{abstract}

\section{Keywords}

Motion-capture, Accuracy, Consistency, Precision, Performance metrics

\section{Introduction}

While there have been several studies examining ball velocity as an outcome metric in baseball pitchers, [1-3] there is a paucity of data on the assessment of accuracy and consistency of pitch location, with only a few parameters at the distal upper extremities examined for pitch accuracy [4-7]. In considering the errors of the over arm throw, Hore, et al. [6] studied the kinematics of finger joints, supporting finger extension timing as a significant variable in prediction of pitch location accuracy. Others have postulated the onset of sequential joint rotations as key components of accuracy in a proximal to distal, temporal fashion [8]. Moreover, Marsh, et al. [4] considered the use of vestibular feedback as a key parameter for pitch location assessments.

Prior studies limit their evaluations to the distal extremity, without considering the entirety of the pitching motion or the position of the pitcher relative to the intended target. In other over arm throwing sports such as cricket, inaccurate pitches were noted to significantly differ by way of lower maximum lead knee lift, decreased knee extension in the arm acceleration phase, significantly less elbow flexion before extension, decreased shoulder external rotation at the beginning of the arm acceleration phase, as well as improper sequence of initiation of pelvic-trunk rotation [5]. In handball, throwing consistency was determined to decrease when throwing to the contralateral side of the throwing arm, while ball velocity was found to be higher when throwing at targets on the ipsilateral side [9].

When referring to consistency of pitch location, one study has evaluated kinematic variables in the creation of a model for baseball pitchers. Glanzer, et al. [10] predicted upper trunk tilt, shoulder abduction, and shoulder horizontal abduction at

*Corresponding author: Joseph E Manzi, Weill Cornell Medical College, New York, NY, USA

Accepted: July 21, 2021

Published online: July 23, 2021

Citation: Manzi JE, Krichevsky S, Roberts N, et al. (2021) Kinematic Models For Pitch Location Metrics in Professional Baseball Pitchers. Arch Sports Med 5(1):247-253 
foot contact as well as shoulder external rotation and shoulder horizontal adduction at maximum shoulder external rotation as the most crucial determinants in pitching consistency. While a major initiating step in assessing this crucial performance metric, this study evaluated a heterogenous cohort of pitchers ranging from youth players to those at the professional level, while only assessing pitching kinematics from foot contact to ball release, not accounting for valuable differences in body positions during the windup and stride phases. Ramappa, et al. [11] has demonstrated even when normalized by body weight, professional pitchers generated significantly greater kinetic values than adolescent pitchers. Additionally, Fleisig, et al. [12] found notable kinematic differences between pitchers of varying age groups as well as playing levels,with older pitchers having shorter stride lengths and more closed pelvis/trunk orientations than their younger counterparts.

Given these inexactitudes, it is important to evaluate player metrics of performance in a homogenous group at a single playing level to truly characterize and substantiate the results observed. Additionally, inclusion of a longer proportion of the total pitching cycle is necessary to capture contributing kinematic factors for accuracy and consistency. Therefore, the purpose of this study was to create a model for consistency and accuracy in pitch location for a uniform, large cohort of professional baseball pitchers utilizing full body, kinematic movements throughout the entirety of the pitching cycle from maximum knee height to maximum shoulder internal rotation. The authors hypothesize parameters of shoulder horizontal adduction and shoulder external rotation at the moment of ball release will be significant predictors for both accuracy and consistency models, given the results of prior studies that have emphasized the importance of shoulder motions in attaining consistency in pitch locations [10].

\section{Materials and Methods}

The study consisted of professional pitchers from 24 distinct teams. Inclusion requirements were that at the time of testing, pitchers were involved in a Major League or Minor League (Low $A$, High $A, A A$, and $A A A$ ) roster and had no record of serious injury (requiring $>2$ weeks of rest or rehabilitation) in the previous six months. As such, each pitcher was medically cleared for throwing in training camp by his team physician. Motus Global (Rockville Centre, NY, USA) de-identified all data prior to distribution and study guidelines were approved by the Institutional Review Board at Hospital for Special Surgery.

Pitching evaluations were performed as previously described [13]. Pitchers reported to the test site where they signed a waiver of privacy and provided consent. Demographic data were reported by the pitcher, including age, preferred throwing arm, experience level, and history of injury. Researcher staff measured and recorded the pitcher's height and weight. The pitcher was given unlimited time to warm up with his preferred routine (e.g., arm bands, stretching, plyometric treatment, and long throttle). Once the pitcher had indicated that he was ready, 46 reflective markers were positioned on anatomical landmarks as previously defined by Luera, et al. [13]. The 8-camera Raptor-E motion analysis system (Motion Analysis Corp, Santa Rosa, CA, USA) was used to collect the location coordinate data of the reflective markers at $480 \mathrm{~Hz}$. Prior to throwing, a single static calibration was obtained, with the pitcher standing still in the capture volume, with legs at hip width apart, shoulders abducted at $90^{\circ}$, and elbows flexed at $90^{\circ}$. The static test was performed to match the pitcher with the laboratory coordinate system and to define the local coordinate systems.

Pitchers were instructed to throw 8 to 12 fastballs with game-like effort to a catcher behind home plate at regulation distance $(18.4 \mathrm{~m})$. Additionally, pitchers were asked to aim for down the middle of the strike zone. Pitchers could pitch at their own set rate, and they were allowed to pitch either from the stretch or the wind-up. Ball speed was collected with a radar gun placed behind the pitcher (Stalker Sports Radar, Richardson, TX, USA). Pitch location was charted from behind the pitcher by a member of the pitching coach staff. Vertical and horizontal coordinates of the ball were manually recorded as they crossed home plate by a member of the players pitching coach staff. One coaching staff personnel would record pitch locations for all pitchers of the same team. All pitch locations were standardized according to the assumption that a typical strike zone is measured by a height of 25.79 " and a width of 17" [14]. The upper-left corner of the strike zone was used as the origin position.

All data processing to develop full body kinematics and throwing arm kinetics was performed in custom MATLAB scripts (The Mathworks, Natick, MA, USA) [13]. A $14 \mathrm{~Hz}$ Butterworth low-pass filter was used to filter the motion data from each marker. Total body segment and joint positions were used to establish key time points of the pitch: maximum knee height $(\mathrm{MKH})$, hand separation (HS), elbow extension $(E E)$, foot contact $(F C)$, maximum shoulder external rotation (MER), ball release (BR), and maximum shoulder internal rotation (MIR) $[13,15]$. FC was identified as the first frame when the lead toe or heel in the $Z$ axis reached the minimum point of contact. BR was calculated as the instant 0.01 seconds after the wrist passed the elbow in the forward direction.

All full body kinematics used in this analysis are as previously described $[13,15]$. Trunk tilt was calculated as an angle from the trunk line (right to left shoulder markers) to the pelvis line (right to left anterior superior iliac spine (ASIS)) in the coronal plane, where negative tilt was towards the glove hand (Figure 1A). Forearm pronation was defined as the rotation of the wrist about the forearm, positive values indicated pronation and negative indicated supination (Figure 1A). Trunk flexion was calculated as the angle between the trunk and pelvis line in the sagittal plane, where a positive value was trunk flexion and negative was extension (Figure 1B). Hip flexion was the angle between the thigh and pelvis, $0^{\circ}$ indicated full extension. Shoulder abduction was calculated as the angle between the upper arm and the line between the two shoulders in the frontal plane, so that the arm straight down against the trunk was $0^{\circ}$ (Figure $1 \mathrm{~B}$ ).

To validate intra-observer reliability, raters were graded on the repeatability of pitch location recordings between all pitchers they observed in a single day. The median absolute 


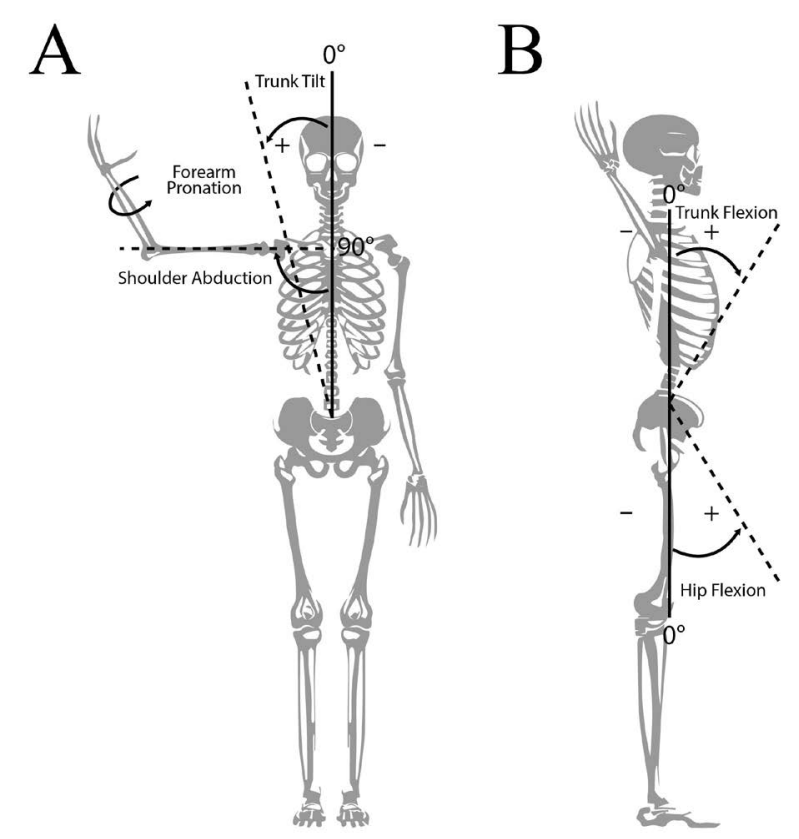

Figure 1: Select kinematic measurements visualized.

deviation (MAD) of accuracy, measured as the normalized absolute center deviation, was calculated for each date, having been validated as a robust measure of variability $[16,17]$. Similarly, MAD was calculated for consistency, measured as the normalized spread of each pitch. Given all pitchers observed by a coach in any single testing day were from the same team, league, and playing level, it could be expected that the accuracy and consistency of all pitchers within this cohort would not have a deviation by more than $20 \%$ averaged among all days. This threshold was set before pitch location metrics were collected. Results showed only $4.2 \%$ of observation dates had an accuracy MAD > 5\% grid length while no test dates had an accuracy MAD $>15 \%$ grid length. Similarly, only $8.8 \%$ of observation dates had a consistency MAD > 5\% grid length while no test dates had a consistency MAD $>15 \%$ grid length. These measurements were well within a reasonable amount of deviation from predicted, demonstrating high intra-rater reliability of the coaching staff for accuracy and consistency.

For each pitcher, the median location across every pitch thrown was calculated. For each pitch thrown, the pitch's Euclidean distance was calculated from (1) the center of the strike zonecoordinate grid and (2) the pitcher's median pitch location. These measurements gave the accuracy and the consistency of the pitch, respectively. Kinematic parameters were estimated from motion capture data averaged for each pitcher. Many of these variables have previously been utilized in analyses of pitching motion $[10,18]$. Stride length in particular, was normalized by pitcher body height.

Elastic net regressions with automated parameter optimization were utilized in assessing all available kinematic variables to model the consistency and accuracy of each pitch and to determine the association of each variable with these two different metrics. Elastic net regression is an extension of the ridge regression and least absolute shrinkage and selection operator (LASSO) regression that combines the regularization techniques of each method and has been shown to have superior performance [19]. Regularized regression models such as elastic net or LASSO have been increasingly used to perform feature selection and assess which variables are most important for a desired biomechanical outcome [20,21]. By introducing a penalization parameter, this model was capable of testing every kinematic variable available, selecting out the ones that were not predictive, there by improving the accuracy of the model predictions. The full dataset was sampled using bootstrapping and cross-validation to test model performance over a series of small subsets of the data.

A random forest model was designed and implemented to assess feature importance [22]. Random forest models are a non-parametric model ensemble method that takes advantage of bagging for regression or classification. A sequence of decision trees attempts to weakly learn the underlying data and these outcomes are aggregated to a single metric. For each tree, the prediction error - mean squared error (MSE) - is recorded. This is also calculated after each independent variable is permuted. The difference between these two measures is averaged across all trees and normalized by the standard deviation of this difference. The contribution of each feature to the MSE is expressed as a percentage and used as a proxy for feature importance. All statistical analyses were performed using $R$ version 3.6.3 (R Foundation for Statistical Computing; Vienna, Austria).

\section{Results}

322 professional pitchers were included in analysis (age: $21.9 \pm 2.1$ years; height: $189.7 \pm 5.7 \mathrm{~cm}$; mass: $94.8 \pm 9.5 \mathrm{~kg}$; ball velocity: $38.4 \pm 1.7 \mathrm{~m} / \mathrm{s}$ ). Derivation of feature importance by random forest regression of pitch accuracy showed that trunk tilt at FC created the largest percent increase of the mean square error (6.6 \% MSE), followed by lead hip flexion at FC (4.2\% MSE), shoulder abduction at FC (4.2\% MSE), trunk tilt at BR (3.8\% MSE), and elbow flexion at MER (Figure 2). The same analysis used to determine pitch consistency showed that trunk tilt at FC had the highest predictive value $13.7 \%$ MSE), followed by trunk flexion at BR (3.3\% MSE), lead foot rotation at $\mathrm{FC}(3.2 \% \mathrm{MSE})$, back hip flexion at FC (2.6\% MSE), and lastly, trunk lateral flexion at FC (2.5\% MSE) (Figure 3). The application of this elastic-net regularized generalized linear model for regressing pitch consistency yielded an $\mathrm{R}^{2}$ value of 0.57 .

Descriptive statistics of predictive parameters shared in the regression models for both accuracy and consistency are listed in addition to average and standard deviation values ( $A$ $\pm S D$ ) of these kinematics among all pitchers: 1) Trunk tilt at FC (A \pm SD: $-7.9 \pm 12.1^{\circ} ; 6.5$ vs. $3.7 \% \mathrm{MSE}$, respectfully), 2) back hip flexion at FC (A \pm SD: $-1.5 \pm 14.4^{\circ} ; 3.4$ vs. $2.6 \% \mathrm{MSE}$, respectfully), 3) lead hip flexion at $F C\left(A \pm S D: 59.6+13.2^{\circ}\right.$; 4.2 vs. $1.1 \% \mathrm{MSE}$, respectfully), 4) shoulder abduction at FC (A \pm SD: $83.9 \pm 11.5^{\circ} ; 4.2$ vs. $1.2 \% \mathrm{MSE}$, respectfully), 5) trunk flexion at $B R\left(A \pm S D: 14.6 \pm 10.0^{\circ} ; 1.9\right.$ vs. $3.3 \% \mathrm{MSE}$, respectfully), and 6) forearm pronation at $B R(A \pm S D: 3.0 \pm$ $16.9^{\circ} ; 2.0$ vs. $1.8 \% \mathrm{MSE}$, respectfully). 
Citation: Manzi JE, Krichevsky S, Roberts N, et al. (2021) Kinematic Models For Pitch Location Metrics in Professional Baseball Pitchers. Arch Sports Med 5(1):247-253

Trunk tilt at FC

Lead hip flexion at FC

Shoulder abduction at FC

Trunk tilt at BR

Elbow flexion at MER

Back hip flexion at FC

Forearm pronation at MER

Back hip flexion at $B R$

Arm slot at BR

Forearm pronation at $\mathrm{BR}$

Trunk flexion at $B R$

Lead hip flexion at BR

Pelvis rotation at FC

Lead hip flexion at MKH

Elbow flexion at $\mathrm{BR}$

Shoulder abduction at BR

Shoulder external rotation at MER

Forearm pronation at MIR

Stride length at FC

Back hip flexion at MKH

Lead foot tilt at FC

Elbow flexion at FC

Shoulder horizonal adduction at MER

Shoulder external rotation at FC

Lead knee flexion at BR

Lead knee flexion at FC

Shoulder horizontal adduction at FC

Lead foot rotation at FC

Trunk lateral flexion at FC

Forearm pronation at FC

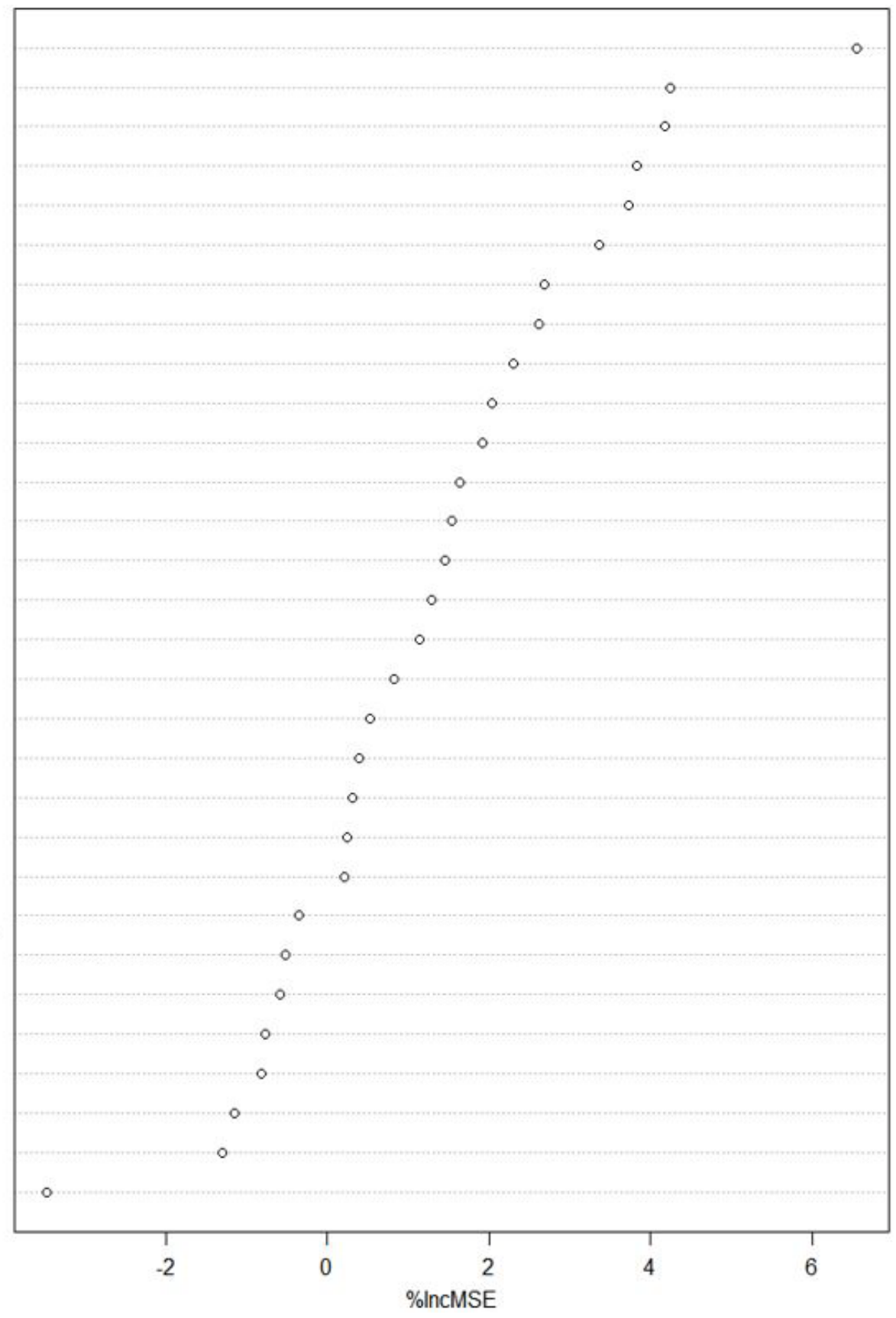

Figure 2: Random forest determined variable importance for pitch accuracy.

Note: FC: Foot Contact; BR: Ball Release; MER: Maximum Shoulder External Rotation; MKH: Maximum Knee Height.

\section{Discussion}

An elastic-net regularized generalized linear model was created to predict accuracy and consistency in pitch location for professional baseball pitchers. Predictive variables shared between the two models included trunk tilt at FC, back hip flexion at FC, lead hip flexion at FC, shoulder abduction at FC, trunk flexion at $\mathrm{BR}$, and forearm pronation at $\mathrm{BR}$. Four out of six of these parameters occurred at FC, while two occurred at BR.

Trunk tilt at FC was a highly predictive parameter in both models for accuracy and consistency, also in agreement with Glanzer et al.'s [10] model for pitch consistency. Furthermore, both leading and back hip flexion at FC were highly predictive variables in both models. Improving pitch location metrics, therefore may be achieved by keeping a more neutral, consistent posture with limited lean away from the pitching arm [23]. Given Robb, et al. [24] demonstrated flexibility of the hip muscles influences pelvis and trunk kinematics during pitching, incorporating training programs to strengthen abdominal core muscles may aid the hip and trunk in retaining a constant posture throughout the pitching cycle. Even more, excessive trunk tilt at BR in high school and collegiate populations was found to be a positive predictor for ball velocity and elbow varus torque (EVT), suggesting trunk tilt has significant influence in other performance metrics of interest as well as a theoretical injury risk profile $[25,23]$. 
Citation: Manzi JE, Krichevsky S, Roberts N, et al. (2021) Kinematic Models For Pitch Location Metrics in Professional Baseball Pitchers. Arch Sports Med 5(1):247-253

Trunk tilt at FC

Trunk flexion at BR

Lead foot rotation at FC

Back hip flexion at FC

Trunk lateral flexion at FC

Forearm pronation at $\mathrm{BR}$

Arm slot at BR

Shoulder abduction at FC

Lead foot tilt at FC

Pelvis rotation at $\mathrm{FC}$

Lead hip flexion at FC

Forearm pronation at MER

Elbow flexion at BR

Lead hip flexion at MKH

Trunk tilt at BR

Shoulder horizontal adduction at FC

Elbow flexion at MER

Forearm pronation at FC

Forearm pronation at MIR

Shoulder external rotation at FC

Back hip flexion at BR

Shoulder abduction at BR

Lead hip flexion at BR

Shoulder external rotation at MER

Shoulder horizonal adduction at MER

Lead knee flexion at BR

Elbow flexion at FC

Stride length at $F C$

Lead knee flexion at FC

Back hip flexion at MKH

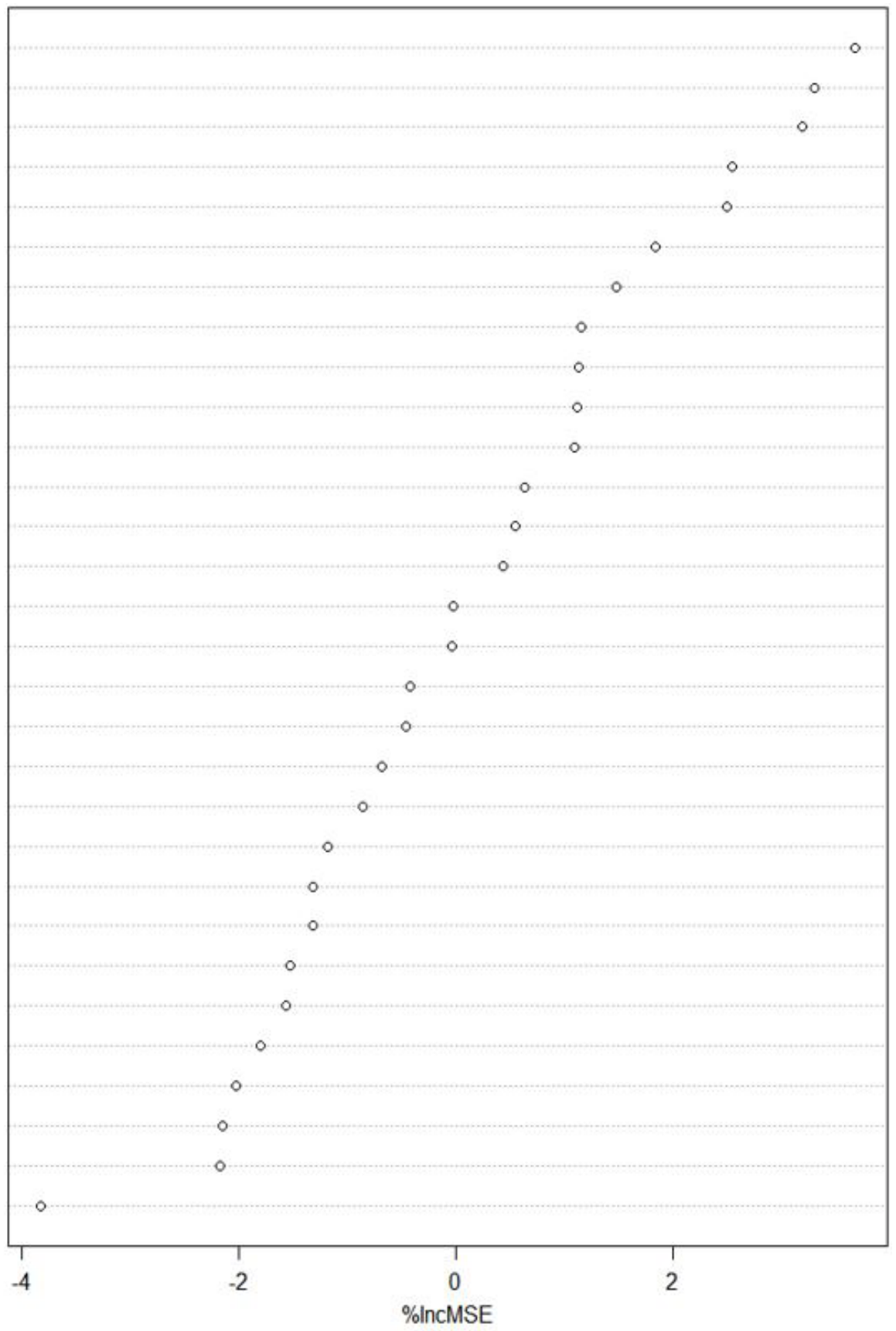

Figure 3: Random forest determined variable importance for pitch consistency.

Note: FC: Foot Contact; BR: Ball Release; MER: Maximum Shoulder External Rotation; MKH: Maximum Knee Height.

The cohort average of this study was found to have shoulder abduction of $83.9 \pm 11.5^{\circ}$ at $\mathrm{FC}$ and $90.6 \pm 8.3^{\circ}$ at BR. Shoulder abduction at FC was deemed a positive predictor for consistency and accuracy models; therefore, achieving repeatable measures in shoulder abduction angles may be beneficial. Prior studies suggest a position of $90^{\circ}$ of shoulder abduction is optimal in maximizing the functional capability of the shoulder in the scapular plane $[26,27]$. If the trunk rotates perpendicular to the longitudinal axis of the arm at the moment of ball release, the longest lever arm is created, which hypothetically results in the fastest ball velocity as well as the greatest functional stability of the shoulder $[26,27]$. Given a relatively constant amount of shoulder abduction occurs throughout the pitching cycle, it is reasonable to assume maintaining this perpendicular position of the shoulder relative to the trunk throughout the pitching cycle may be beneficial for performance outcomes of consistency and accuracy.

The degree of forearm pronation at BR is highly predictive of ball consistency and accuracy, given this is the initial moment the hand separates entirely from the ball. Furthermore, forearm pronation at BR is also the closest upper extremity parameter measured relative to the ball. Maintaining a relatively constant amount of pronation between pitches may in fact be a way to improve pitcher consistency. This 
finding also raises the question of if a limitation of this study is not assessing more fine-tune body kinematics at the wrist, hand, and fingers in addition to the forearm in evaluations of accuracy and consistency. This may also be why only a small amount of variance was predicted for the accuracy model. Indeed, finger extension timing as well as the sequential joint rotations of the elbow,wrist and fingers, have all previously shown to be positive predictors for pitch location accuracy $[6,8]$. Further kinematic studies should include additional markers to evaluate wrist as well as finger temporal and positional motions throughout the pitch cycle to potentially better characterize accuracy as a performance outcome.

There are several additional limitations of this study. Pitch locations were determined by a member of the coaching staff. While this could introduce error in measuring the accuracy of pitch locations, it is less likely to have a negative impact on the consistency given that the same person evaluated all of the pitches for a given pitcher. Another limitation is that only fastballs were used in this study making the generalizability of these findings to other pitch types uncertain. Lastly, these metrics were collected in the span of 8-12 pitches in a lowpressure scenario that does not realistically characterize a game-like setting where factors like pitch count, fatigue, and player psyche all can influence a pitchers ability to throw with consistency and/or accuracy [28,29].

Creating a model in professional baseball pitchers based on kinematic variables was able to characterize a majority of the variance for pitch location consistency. Four out of the top six parameters found to be predictive in models for accuracy and consistency involved variance at the hip and trunk as well as foot contact as the most critical moment. In order to maximize their ability to achieve target location of their pitches, pitchers should strive for consistency in hip and upper torso motions, particularly at foot contact.

\section{References}

1. Urbin MA, Fleisig GS, Abebe A, et al. (2013) Associations between timing in the baseball pitch and shoulder kinetics, elbow kinetics, and ball speed. Am J Sports Med 41: 336-342.

2. Post EG, Laudner KG, McLoda TA, et al. (2015) Correlation of shoulder and elbow kinetics with ball velocity in collegiate baseball pitchers. J Athl Train 50: 629-633.

3. Crotin RL, Bhan S, Karakolis T, et al. (2020) Fastball velocity trends in short-season minor league baseball. J Strength Cond Res 27: 2206-2212.

4. Marsh DW, Richard LA, Williams LA, et al. (2020) The relationship between balance and pitching error in college baseball pitchers. J Strength Cond Res 18: 441-446.

5. Sachlikidis A, Salter C (2007) A biomechanical comparison of dominant and non-dominant arm throws for speed and accuracy. Sport Biomech 6: 334-344.

6. Hore J, Watts S, Tweed D (1996) Errors in the control of joint rotations associated with inaccuracies in overarm throws. J Neurophysiol 75: 1013-1025.

7. Hore J, Watts S, Tweed D (1999) Prediction and compensation by an internal model for back forces during finger opening in an overarm throw. J Neurophysiol 82: 1187-1197.
8. Herring RM, Chapman AE (1992) Effects of changes in segmental values and timing of both torque and torque reversal in simulated throws. J Biomech 25: 1173-1184.

9. van den Tillaar R (2020) The effects of target location upon throwing velocity and accuracy in experienced female handball players. Front Psychol 11: 2006.

10. Glanzer JA, Diffendaffer AZ, Slowik JS, et al. (2019) The relationship between variability in baseball pitching kinematics and consistency in pitch location. Sport Biomech.

11. Ramappa AJ, Chen PH, Hawkins RJ, et al. (2010) Anterior shoulder forces in professional and little league pitchers. J Pediatr Orthop 30: 1-7.

12. Fleisig GS, Barrentine SW, Zheng N, et al. (1999) Kinematic and kinetic comparison of baseball pitching among various levels of development. J Biomech 32: 1371-1375.

13. Luera MJ, Dowling B, Muddle TWD, et al. (2020) Differences in rotational kinetics and kinematics for professional baseball pitchers with higher versus lower pitch velocities. J Appl Biomech.

14. (2021) Prospectus feature: The universal strike zone. Baseball prospectus.

15. Luera MJ, Dowling B, Magrini MA, et al. (2018) Role of rotational kinematics in minimizing elbow varus torques for professional versus high school pitchers. Orthop J Sport Med 6: 2325967118760780 .

16. Stuart M, Hoaglin DC, Mosteller F, et al. (1984) Understanding robust and exploratory data analysis. Stat 33: 320.

17. Croux C, Dehon C (2014) Robust estimation of location and scale. John Wiley \& Sons, Ltd, Chichester, UK.

18. Dowling B, Laughlin WA, Gurchiek RD, et al. (2020) Kinematic and kinetic comparison between American and Japanese collegiate pitchers. J Sci Med Sport 23: 1202-1207.

19. Gonzales GB, De Saeger S (2018) Elastic net regularized regression for time-series analysis of plasma metabolome stability under sub-optimal freezing condition. Sci Rep.

20. Joodaki H, Gepner B, Kerrigan J (2020) Leveraging machine learning for predicting human body model response in restraint design simulations. Comput Methods Biomech Biomed Engin 24: 597-611.

21. Peterson Silveira R, Stergiou P, Figueiredo P, et al. (2018) Key determinants of time to $5 \mathrm{~m}$ in different ventral swimming start techniques. Eur J Sport Sci 18: 1317-1326.

22. Bharathidason S, Jothi Venkataeswaran C (2014) Improving classification accuracy based on random forest model with uncorrelated high performing trees. Int J Comput Appl 101: 26-30.

23. Solomito MJ, Garibay EJ, Nissen CW (2018) Sagittal plane trunk tilt is associated with upper extremity joint moments and ball velocity in collegiate baseball pitchers. Orthop J Sport Med 6: 2325967118800240.

24. Robb AJ, Fleisig G, Wilk K, et al. (2020) Passive ranges of motion of the hips and their relationship with pitching biomechanics and ball velocity in professional baseball pitchers. Am J Sports Med 38: 2487-2493.

25. Oyama S, Yu B, Blackburn JT, et al. (2013) Effect of excessive contralateral trunk tilt on pitching biomechanics and performance in high school baseball pitchers. Am J Sports Med 41: $2430-2438$. 
26. Matsuo T, Matsumoto T, Mochizuki Y, et al. (2020) Optimal shoulder abduction angles during baseball pitching from maximal wrist velocity and minimal kinetics viewpoints. J Appl Biomech 18: 306-320.

27. Escamilla RF, Fleisig GS, Barrentine SW, et al. (2020) Kinematic comparisons of throwing different types of baseball pitches. J Appl Biomech 14: 1-23.
28. Yang SC, Wang CC, Lee S DA, et al. (2016) Impact of 12-s rule on performance and muscle damage of baseball pitchers. Med Sci Sports Exerc 48: 2512-2516.

29. Whiteside D, Martini DN, Zernicke RF, et al. (2016) Changes in a starting pitcher's performance characteristics across the duration of a major league baseball game. Int J Sports Physiol Perform 11: 247-254. 